\title{
Desarrollo de Aplicaciones Utilizando Geoservicios de una Infraestructura de Datos Espaciales. Casos de éxito: Directorio Comercial SC, AgroMAG, IDEHN Mobile Developing Applications using Spatial Data Infrastructure GeoServices. Use Cases: Directorio Comercial SC, AgroMAG, IDEHN Mobile
}

Oscar Víquez-Acuña', Leonardo Víquez-Acuña², Marlen Treviño-Villalobos ${ }^{3}$, Marcela Chaves-Álvarez ${ }^{4}$

Víquez-Acuña, O; Víquez-Acuña, L; Treviño-Villalobos, M; Chavez-Álvarez, M. Desarrollo de Aplicaciones Utilizando Geoservicios de una Infraestructura de Datos Espaciales. Casos de éxito: Directorio Comercial SC, AgroMAG, IDEHN Mobile. Tecnología en Marcha. Vol. 30-3. Julio-Setiembre 2017. Pág 85-96

DOI: 10.18845/tm.v30i3.3275

1 Instituto Tecnológico de Costa Rica. Carrera de Computación. San Carlos, Costa Rica. Correo electrónico: oviquez@tec.ac.cr.

2 Instituto Tecnológico de Costa Rica. Carrera de Computación. San Carlos, Costa Rica. Correo electrónico: Iviquez@tec.ac.cr.

3 Instituto Tecnológico de Costa Rica. Carrera de Computación. San Carlos, Costa Rica. Correo electrónico: mtrevino@tec.ac.cr.

4 Instituto Tecnológico de Costa Rica. Carrera de Computación. San Carlos, Costa Rica. Correo electrónico: mchaves@tec.ac.cr. 


\section{Palabras clave}

Infraestructura de Datos Espaciales; IDE-HN, WMS; WFS; Geoserver; PostGis; Android; Leaflet.

\section{Resumen}

Las Infraestructuras de Datos Espaciales (IDE) permiten primordialmente la administración y publicación de información geográfica, ya sea de índole nacional, regional o local, logrando con esto el potencial establecimiento de una red de nodos de diversos actores proveedores de datos que permita la interrelación de información. En este contexto, la IDE Huetar Norte de Costa Rica (IDEHN) se creó con el propósito de proveer información regional de diversas fuentes.

Para la consulta de esta información, la IDEHN facilita el uso de geoservicios que permiten el acceso remoto de los datos a través de geoportales, herramientas para Sistemas de Información Geográficos e inclusive a través de aplicaciones de software a la medida para escritorio o dispositivos móviles.

En relación con este espacio de trabajo de desarrollo de aplicaciones a la medida, este artículo presenta tres casos de éxito en el uso de geoservicios proporcionados por la IDEHN para gestionar información geográfica en dispositivos móviles.

\section{Abstract}

A Geographic Data Infrastructure (GDI) allow users to manage and publish geographic data representing national, regional and local information from several nodes. These nodes can establish a GDI network if the information they contain is related to each other. In this context, the Geographical Data Infrastructure for the Huetar Norte región (IDEHN) was created to publish regional information from various sources.

To consult this information, IDEHN makes available geoservices to get remote access to the data from geoportals, geographical information systems tools and specific desktop software or mobile devices apps.

Over this workspace, this article presents three software examples of using IDEHN geoservices to access geographical information in mobile devices.

\section{Keywords}

Geographic Data Infrastructure; IDE-HN; WMS; WFS; Geoserver; PostGis; Android; Leaflet.

\section{Introducción}

En la actualidad hay muchas definiciones sobre el concepto de Infraestructura de Datos Espaciales (IDE). Como información relevante, este término fue considerado por primera vez en 1993 por el U.S. National Research Council para referirse a un marco de tecnologías, políticas y disposiciones institucionales que trabajando conjuntamente, facilitan la creación, el intercambio y el uso de los datos geoespaciales y recursos de información relacionados a través de una comunidad de intercambio de información [1].

También, Kuhn [2] define la IDE como “...una serie de acuerdos acerca de estándares tecnológicos, acuerdos institucionales y políticas que permiten el descubrimiento y uso de 
información geoespacial por parte de usuarios y con propósitos diferentes de aquellos para los que fue creada esa información". Si se analizan las definiciones anteriores, se pueden extraer algunos rasgos comunes como son los términos: información espacial, disponibilidad, intercambio y/o interoperabilidad de la información.

Una IDE está conformada por cuatro componentes fundamentales: los datos, los metadatos, los servicios y la organización, como se muestra en la figura 1. Los datos son una representación simbólica (numérica, alfabética, algorítmica etc.), un atributo o una característica de una entidad. Los metadatos son la información que describe los conjuntos y servicios de datos espaciales. La organización es el componente más complejo y hace que el resto funcione y se mantenga, incluye el personal humano, los estándares y normas que hacen que los sistemas puedan interoperar, las reglas y acuerdos entre los productores de datos, y finalmente están los servicios que son las funcionalidades accesibles mediante los recursos de Internet [3]. Entre los anteriores podemos encontrar el servicio de consultas de mapas en formato de imagen (WMS - Web Map Service) [11], el servicio de descarga de datos geográficos con todos sus atributos (WFS - Web Feature Service) [12], el servicio de acceso a datos de cobertura ráster fotográficas (WCS - Web Coverage Service), entre otros.

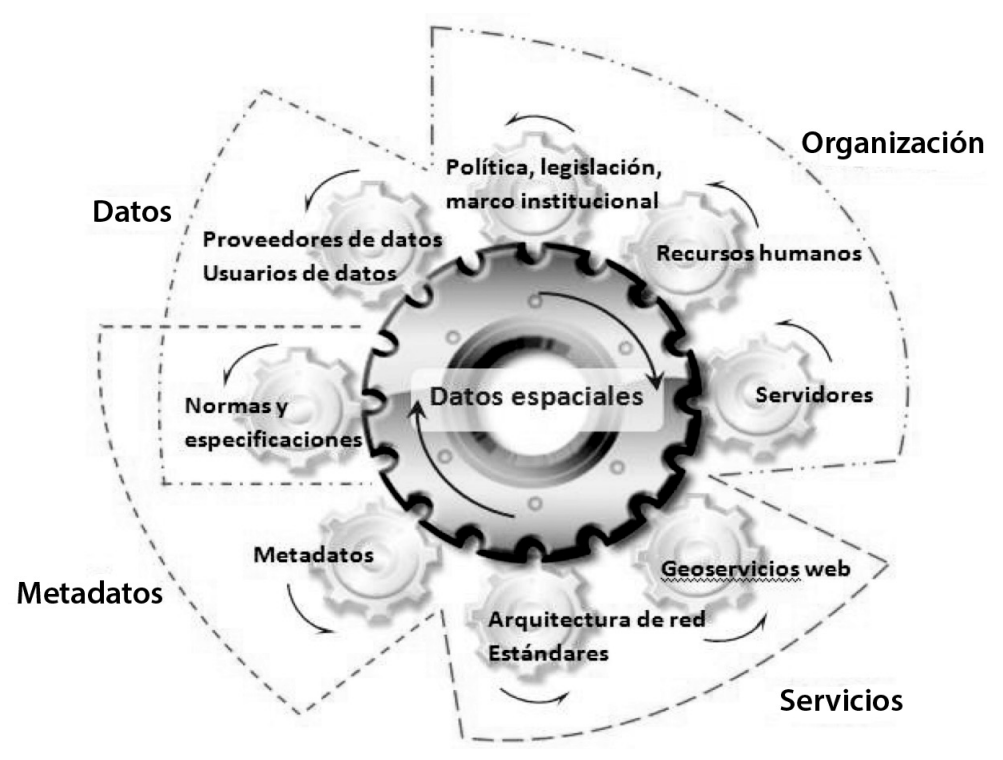

Figura. 1. Componentes fundamentales de una IDE. Tomado de [5].

Al mismo tiempo se puede mencionar que una IDE, a nivel técnico, está compuesta por elementos de hardware tales como servidores con alto poder de cómputo, almacenamiento de la información y conectividad, así como elementos de software necesarios para el acceso a la información. Aplicaciones como visualizadores de mapas, servicios de transferencia de información, gestores de datos geográficos, búsquedas a partir de catálogos de metadatos, entre otros, son ejemplos de herramientas que deben estar presentes en una IDE. Para ello, las opciones de software libre son una seria alternativa para el establecimiento rápido y accesible de estas aplicaciones [4].

En relación con el área geográfica que abarcan, las IDE forman una estructura escalonada en diferentes niveles en función de la extensión y de la información geográfica que gestionan. Los niveles superiores integran a los inferiores, de tal forma que las IDE se pueden clasificar en niveles: continental, nacional, regional y local (ver figura 2). 
En este contexto, la Infraestructura de Datos Espaciales del Estado Plurinacional de Bolivia [6], en el documento "Requisitos técnicos para implementar un nodo", define a un nodo de IDE, como una plataforma informática que provee por lo menos un catálogo de metadatos, autónoma de los otros nodos de la IDE a nivel de los datos, metadatos y eventualmente en aspectos tecnológicos.

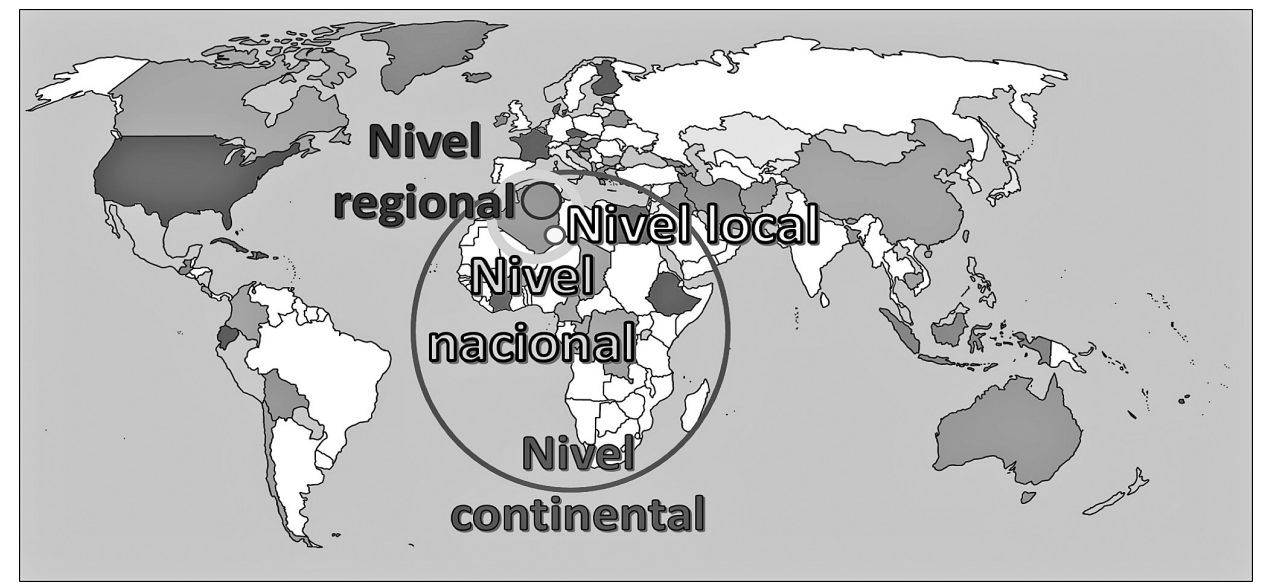

Figura. 2. Niveles organizativos de una IDE. Tomado de [5].

Las funciones principales de un nodo son:

- Almacenamiento de los metadatos en un catálogo.

- Almacenamiento de datos geográficos (opcional).

- Servicios web para el acceso en lectura y escritura (opcional) a los datos y metadatos.

En Costa Rica, estos niveles de IDE se ven reflejados inicialmente a nivel nacional con el Sistema Nacional de Información Territorial (SNIT por sus siglas y disponible en www.snitcr.go.cr ) que funge como ente rector de las políticas de manejo de información geográfica estandarizada. A partir del nivel nacional, pueden surgir IDEs regionales y locales (cantonales o distritales). Un ejemplo de nodo regional lo constituye, en la Región Huetar Norte, la IDEHN (www.idehn. tec.ac.cr). En este artículo se detallan los trabajos elaborados por la IDEHN, principalmente en temas de desarrollo de aplicaciones asociadas a los datos que provee la plataforma, y el ejemplo a nivel cantonal de la plataforma IDESCA (www.idesca.cr), desarrollada para el manejo de la información del Gobierno Local de San Carlos.

La IDEHN brinda los servicios de manejo de información estandarizada para acceso público y privado. Dicha información puede ser consultada y utilizada directamente en el portal de la plataforma o puede ser exportada a diversos formatos para diferentes usos. Una de las particularidades que se pueden obtener de estos servicios es la posibilidad de crear aplicaciones particulares que respondan a múltiples fines y que para ello utilicen esta información georeferenciada.

En este contexto, se han implementado una serie de aplicaciones que hacen uso de estos servicios de acceso a datos, los cuales demostrando su potencialidad, lo cual plantea el reto de desarrollar otras herramientas con finalidades similares. Por lo tanto, el presente trabajo tiene como propósito mostrar los resultados preliminares en el desarrollo de tres aplicaciones particulares denominadas: Directorio Comercial San Carlos (DCSC), AgroMAG e IDEHN Mobile. 


\section{Geoservicios WMS y WFS de la IDE-HN.}

En este momento, la IDEHN trabaja en el proceso de publicación de capas de datos geográficos con sus respectivos metadatos. A la fecha de elaboración de este documento se han publicado un total de 45 capas de datos, con sus respectivos metadatos estandarizados según normas nacionales (como se muestra en la figura 3). Entre las capas de datos publicadas se encuentra: cantones, distritos, poblados, volcanes, cerros, red vial, ríos, calles, curvas de nivel, entre otras. Además, se han elaborado 6 mapas temáticos.

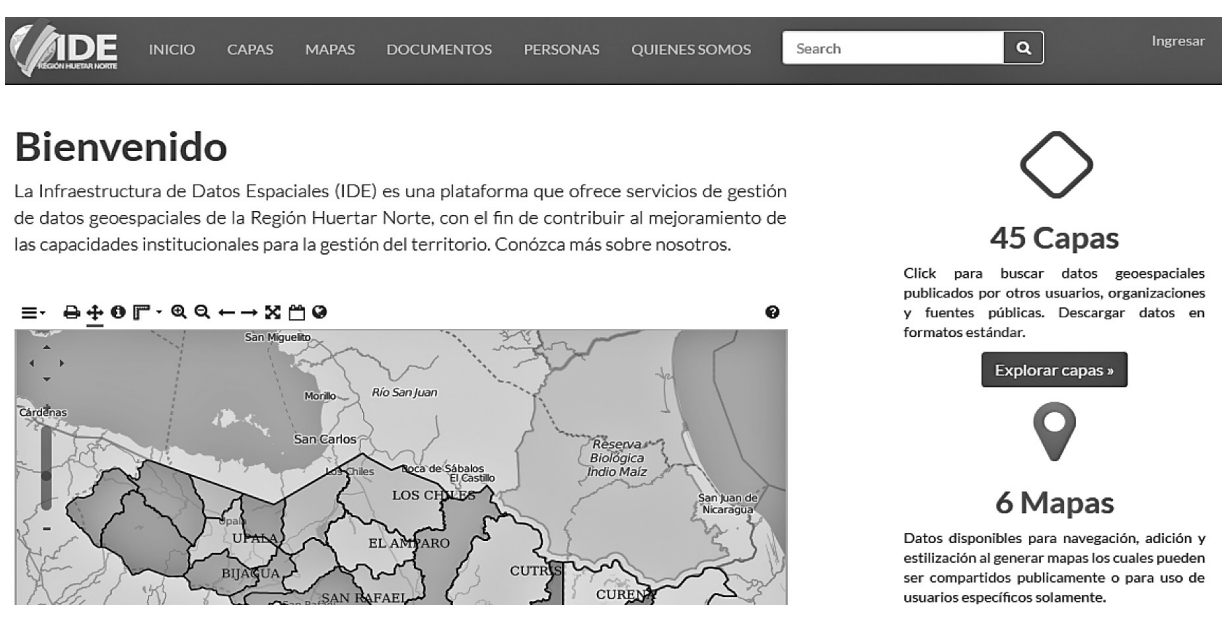

Figura. 3. Geoportal IDEHN.

El geoservicio WMS de la IDEHN provee una interfaz HTTP para acceder a imágenes georeferenciadas de distintas bases de datos geoespaciales. Estas imágenes asocian un conjunto específico de datos, previamente configurados, que le permiten al usuario reconocer las características observables de los elementos presentes en la imagen, tanto en clientes web como en clientes móviles, como teléfonos inteligentes y tabletas.

En la figura 4, se muestra la capa de datos de curvas de nivel cada 200 metros para la región Huetar Norte de Costa Rica a escala 1:50000, producida por el Instituto Geográfico Nacional. Ésta es mostrada a través del geoservicio WMS, en el visualizador OpenLayers con interfaz sencilla. Se puede apreciar, además, la consulta de varios puntos específicos y su despliegue particular en este visor web minimalista.

El geoservicio WFS de la IDEHN por su parte permite principalmente descarga de capas vectoriales donde se incluye su geometría y las tablas de atributos asociadas que posteriormente pueden ser exportadas a otros formatos como Shapefile, Geography Markup Language (GML), etc. Todo esto a través del lenguaje de marcas extensible (XML, por sus siglás en inglés). Normalmente este servicio puede implicar amplios volúmenes de tráfico de información, lo que ocasiona sea más utilizado por clientes de manejo de datos geográficos como ArcGIS, QGIS, gvSIG, etc; pero al mismo tiempo puede utilizarse en otros procesos que requieran del acceso a los datos de forma directa.

\section{Desarrollo de aplicaciones utilizando geoservicios}

Para el desarrollo de las aplicaciones implementadas por la IDEHN, fue necesaria la utilización de las herramientas tecnológicas que mejor se adaptaron a las condiciones existentes, tanto 
para el desarrollo nativo en plataformas de Android, junto con componentes web específicos. En el siguiente apartado, se mencionan las principales herramientas utilizadas para el desarollo de las aplicaciones Directorio Comercial SC, AgroMAG e IDEHN Mobile.

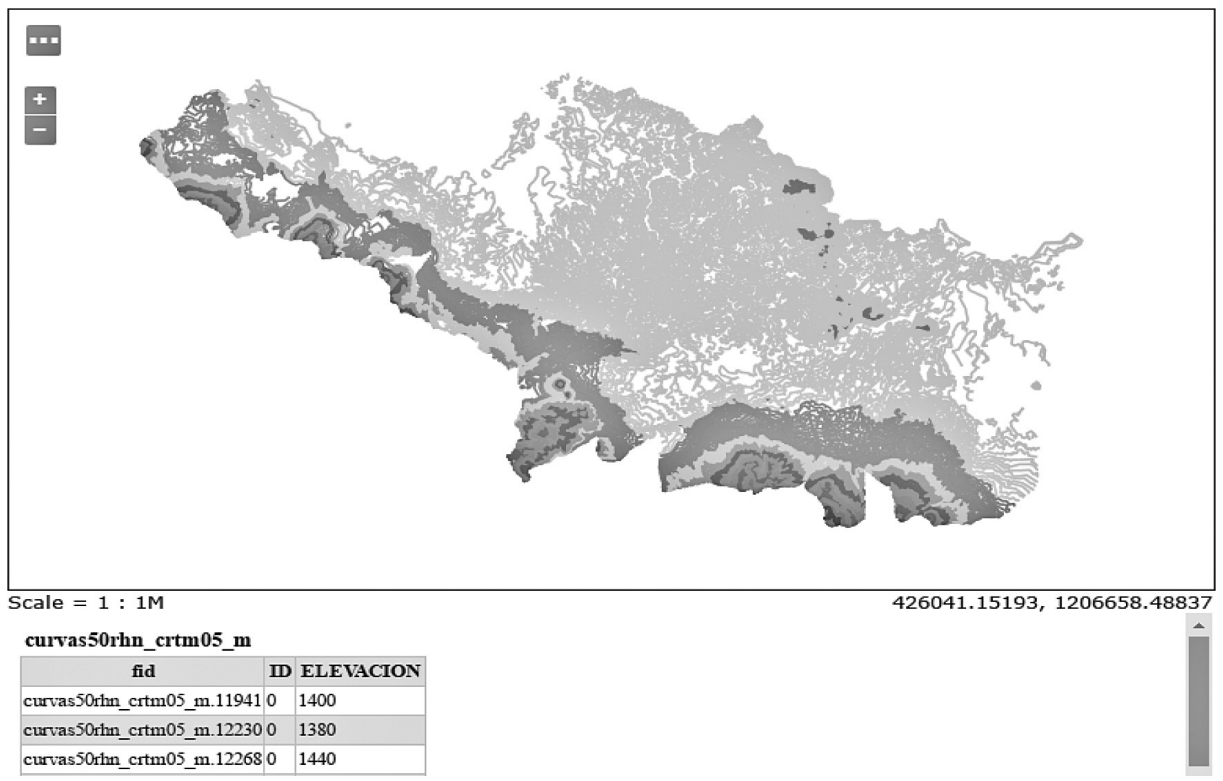

Figura. 4. Curvas de Nivel RHN, escala 1:50000 en visor OpenLayer utilizando WMS de la IDEHN.

\section{Tecnologías Involucradas}

Las principales tecnologías involucradas para el desarrollo de las aplicaciones que utilizan geoservicios son:

- Android Studio: es un entorno de desarrollo integrado para la plataforma Android que también utiliza herramientas del kit de desarrollo de software (SDK, por sus siglas en inglés) de Java. La creación de la interfaz se define como nativa, utilizando luego JavaScript para el manejo de las estructuras y la lectura del servicio WMS [6].

- OpenStreetMap: es un proyecto colaborativo y se utiliza como base para la creación de mapas libres y editables [7].

- Librería Leaflet: es una librería de código abierto de JavaScript para la construcción de mapas en la web. Es compatible con la mayoría de plataformas móviles y de escritorio, posee soporte para HTML5 y CSS3. Además, permite a los desarrolladores, sin necesidad de usar un Sistema de Información Geográfico (SIG), visualizar muy fácilmente mapas web alojados en un servidor público [8].

- PostgreSQL: Motor de bases de datos open source para el manejo de bases de datos relacionales [10].

- PostGIS: Extensión para el motor de bases de datos PostgreSQL que brinda soporte para el menejo de datos geográficos vectoriales y ráster [9].

Con la implementación de la IDEHN y los servicios geográficos ofrecidos por la plataforma se ha tenido la necesidad de expandir los métodos de acceso a la información, por medio del desarrollo de aplicaciones para dispositivos móviles (app). A la fecha se han tenido resultados 
preliminares en el desarrollo de tres aplicaciones particulares denominadas: Directorio Comercial San Carlos (DCSC), AgroMAG e IDEHN Mobile.

El Directorio Comercial San Carlos consiste en una aplicación desarrollada para la Municipalidad de San Carlos que permite el despliegue de información relacionada con los patentados registrados de dicho cantón, específicamente los detalles generales de contacto así como el componente geográfico. La aplicación incorpora además la funcionalidad de cálculo de rutas desde el lugar donde se encuentre el usuario hacia el punto específico seleccionado.

La aplicación AgroMAG es un prototipo creado para el manejo de fincas, en la región Huetar Norte, que trabajan bajo el régimen de protección ambiental y cambio climático. Estas fincas deben ser monitoreadas por funcionarios del MAG, por lo que la aplicación sirve de puente entre el dueño de la finca que registra los cambios en la misma y el técnico del MAG que monitorea dichos avances, hacia el logro de los objetivos particulares planteados.

Por último, la aplicación IDEHN Mobile fue diseñada para brindarle al usuario del geoportal IDEHN una perspectiva de manejo de las capas de datos que pueden encontrarse en la plataforma, desde su teléfono celular con el sistema operativo Android. Es posible, desde la aplicación, cargar las capas de datos de la IDEHN así como de otros repositorios y consultar metadatos e información detallada de cada una de ellas y de sus componentes geográficos.

Acceso a la información geográfica

El soporte de datos para las aplicaciones desarrolladas se fundamenta en el consumo directo de los geoservicios que ofrece el geoportal o bien el acceso directo a las bases de datos que dan soporte a la información geográfica.

Con el desarrollo de la app IDEHN Mobile se ha realizado una implementación manual del protocolo WFS para leer las distintas características de los objetos geográficos de modo que se pueda mostrar su representación geométrica y los datos descriptivos vinculados. El procolo WFS es implementado con un protocolo de comunicación HTTP para la tranferencia de datos XML estandarizados por el consorcio OGC (www.opengeospatial.org), y denominado GML; que brinda la gramática necesaria para la representación de objetos geográficos [13].

Los datos geográficos están almacenados y controlados por un servidor PostgreSQL y soportados por la extensión PostGIS, desde donde son accedidos por un servidor web a través del procoloclo WFS, este servicio envía paquetes de contenido GML y la app toma estos paquetes y los interpreta para la visualización tanto de los objetos geográficos como de la información descriptiva (ver figura 5).

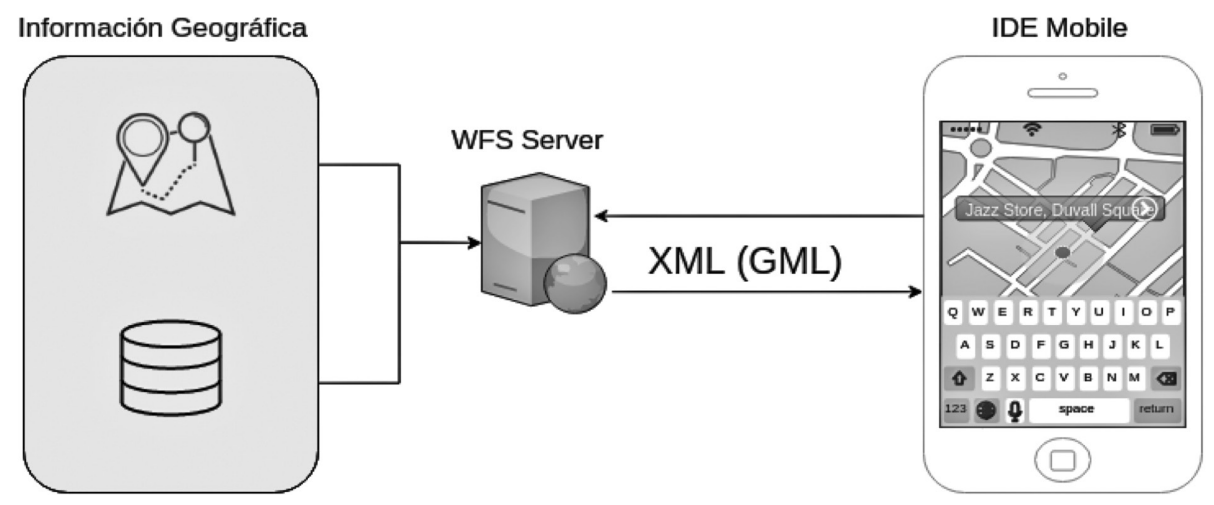

Figura. 5. Modelo de transferencia de datos utilizando el protocolo de comunicación WFS 
Por otro lado, las aplicaciones Directorio Comercial San Carlos y AgroMAG leen los datos geográficos directamente desde las bases geográficas, lo que permite un mayor control de los datos y su manipulación.

La app Directorio Comercial logra con este tipo de conexión, mantener puntos geográficos de referencia de los locales comerciales del cantón de San Carlos, al mismo tiempo que los asocia con información descriptiva y archivos multimedia, en tablas distintas a los datos geográficos. Sin embargo, estos datos siempre se encuentran asociados por conexiones relacionales. Se ha logrado con esto realizar una base de datos híbrida con las funcionalidades relacionales asociadas, el funcionamiento de la aplicación y el manejo de datos geográficos. La figura 6 representa la implementación a la conexión con los datos.

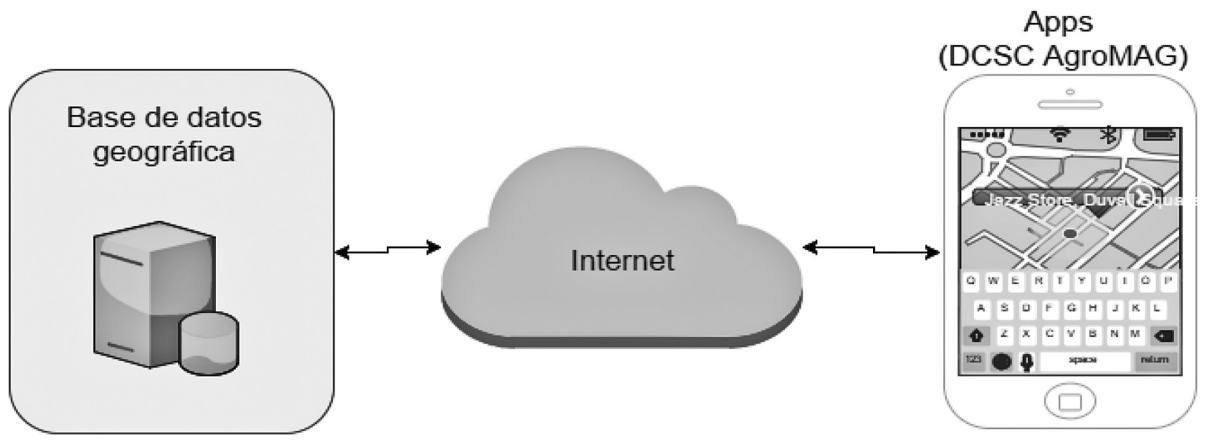

Figura. 6. Conexión directa a base de datos geográfica.

Del mismo modo, la app AgroMAG establece una conexión directa con la base de datos, pero el manejo de los datos geográficos tiene un mayor grado de relevancia pues es fundamental el registro histórico de la información tanto geográfica como descriptiva. AgroMAG almacena información del uso de los apartos de las fincas por lo que es requerido conocer la historia de uso de los mismos, tomando en consideración que estas segmentaciones de fincas también pueden ser dinámicas en el tiempo.

La figura 7 muestra un subesquema de base de datos, el cual detalla la solución planteada para el modelo relacional, en donde la finca está conformada por una serie de apartos geográficos que cuentan con una relación dirigida a sí mismos para determinar el aparto que lo antecede (si existiera). Cada cambio o creación de apartos genera un nuevo estado final de las fincas que se registra en una tabla que construye el histórico, de este modo siempre se consulta la última modificación y es posible navegar en estados históricos tanto a nivel de finca como a nivel de apartos.

Es importante recalcar que tanto las tablas de fincas y de apartos contienen atributos de tipo Geometry, con los cuales se realizan geoprocesos para la creación de las versiones finales de las fincas y para representar los cambios topológicos históricos.

\section{Despliegue de información geográfica}

El despliegue de información geográfica fuera del ámbito de la plataforma IDE presenta un reto particular en el desarrollo de las aplicaciones, ya que depende de los requerimientos específicos de cada aplicación y del geoservicio que se utilice para obtener los datos. Por ejemplo, si se desea desplegar los datos a partir del WMS, se hará uso de algún visor que sea complatible con el tipo de aplicación que se esté desarrollando, sea esta nativa de algún dispositivo móvil, o del ámbito de las aplicaciones web. Por el contrario, si se hace referencia al 
uso del servicio WFS brindado por la IDEHN, el despliegue debe ser diferente y las tecnologías utilizadas deben adaptarse para dicho fin.

En el caso particular del Directorio Comercial, el despliegue de datos geográficos se realiza a partir de una capa de datos de la IDE que es transformada a formato GeoJSON; para luego ser desplegada en cualquier visualizador web por medio de la librería LeafLet utilizando una plantilla denominada Bootleaf para el despliegue elegante de datos masivos. Concretamente, lo anterior potenció el despliegue eficiente, tanto en aplicaciones web de uso general, como en visualizadores nativos de Android con recursos limitados, tanto de hardware, como de acceso a Internet. En la figura 8, se muestra el despliegue de una capa de datos de la IDEHN, tanto en un navegador web robusto como en un visualizador web nativo en Android, y en dicha imagen se puede apreciar que ambos navegadores presentan resultados similares en términos de eficiencia.

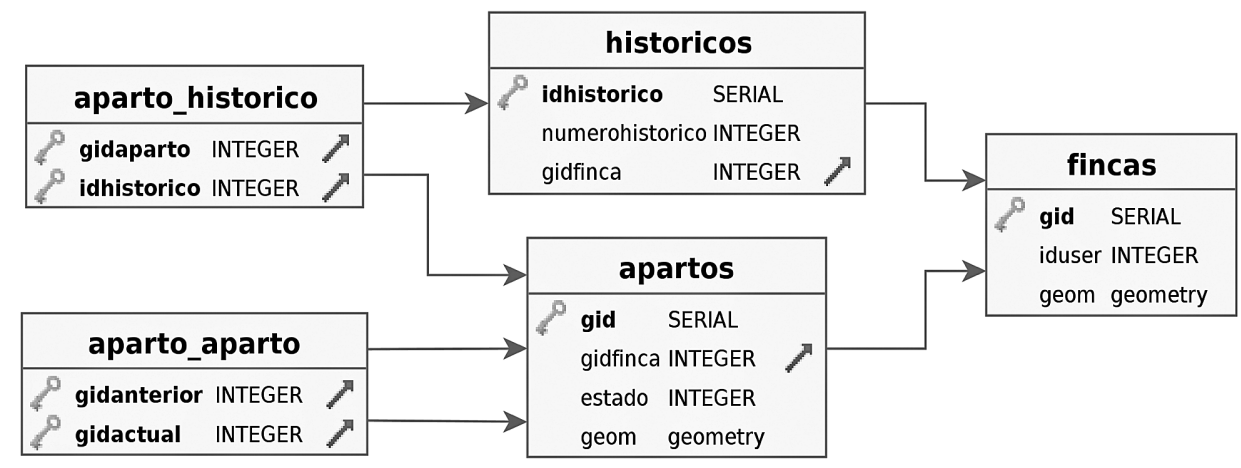

Figura. 7. Modelo relacional para el manejo de datos geográficos históricos.
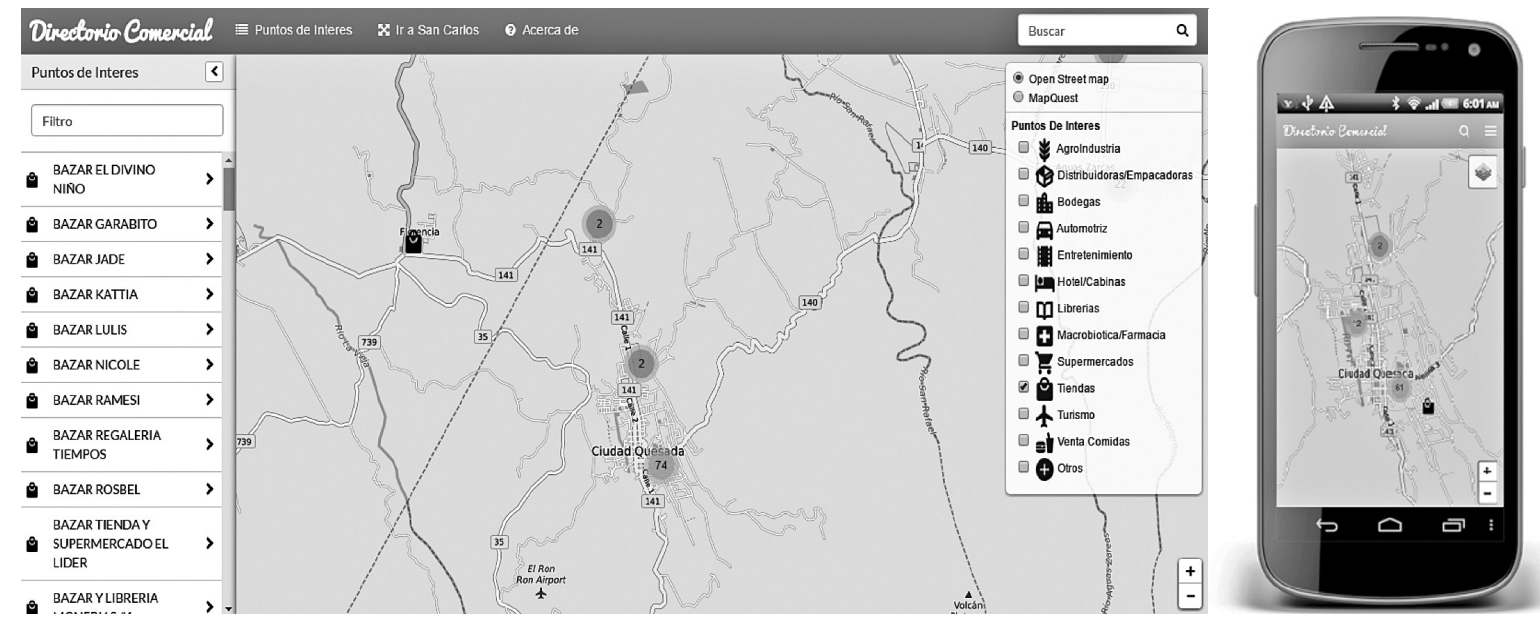

Figura. 8. Bootleaf y LeafLet desplegando información del Directorio Comercial desde diversas plataformas.

Es importante mencionar, que la aplicación nativa de Android para el despliegue de las capas de datos de la plataforma accede a información más detallada, como es el caso de los metadatos de las capas y los campos de los objetos geográficos. Para esto accede directamente al geoservicio WFS pero a través de los recursos públicos que se encuentran en 
formato XML, a partir de los que es posible obtener todos los datos mencionados de las capas que así lo permitan.

Este proceso de reconocimiento y análisis del XML conlleva un procesamiento mayor, sobre todo para un dispositivo móvil, por lo que el desempeño cuando la capa de datos contiene una cantidad significativa de información se podría ver comprometido sin llegar a dejar de mostrar los datos. Por este motivo, el despliegue se realizó también utilizando la librería Leafleat (ver figura 9).

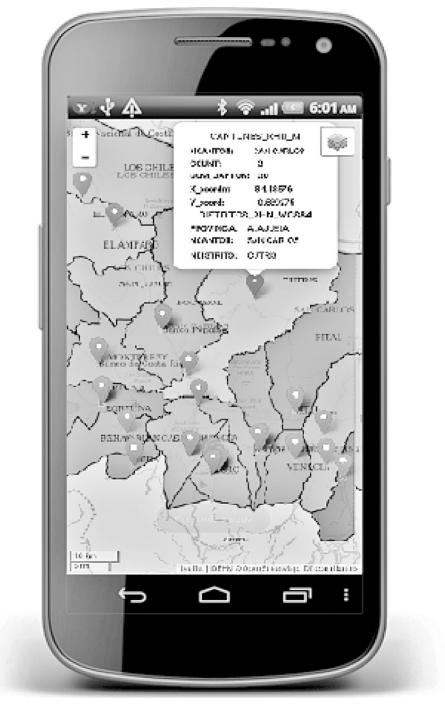

Figura 9. Aplicación IDEHN Mobile desplegando datos de la plataforma mediante Booleaf en dispositivo Android.

\section{Conclusiones}

La IDEHN, ha logrado reunir fuentes de información relevante para la toma de decisiones de la Región Huetar Norte (Costa Rica), definida como su área de influencia. Se ha logrado vincular distintos actores con información geográfica que en este momento están viviendo una transformación en su esquema de manejo de datos geográficos y su libre publicación para el ofrecimiento de geoservicios. Este cambio, apunta a la necesidad de modificar el modelo de retención de la información para uso interno y sacar un mayor provecho, ofreciendo servicios con esta información para ser demandados por portales geográficos, sistemas de información geográficos de escritorio o apps para dispositivos móviles.

Lo anterior, nos permite determinar que una de las principales potencialidades que brindan las IDE y especialmente sus geoservicios, es el desarrollo de aplicaciones de terceros que consuman los recursos que se brindan. Aunado a ello, se tiene que la información geográfica especializada que puede proveer una IDE, permite el despliegue de capas actualizadas que brindan información valiosa, para ser utilizada con múltiples propósitos y que pueden potenciar el desarrollo de aplicaciones principalmente web o para dispositivos móviles.

Por otro lado, el desarrollo de este tipo de aplicaciones permite a usuarios no especializados tener un mejor conocimiento del ámbito geográfico y cartográfico de su territorio; ya que se pone a disposición de éstos información geográfica actualizada en forma permanente y de fácil 
manejo. Estas caraterísticas representan un avance sustancial en relación al tipo de usuarios que requiere un software SIG tradicional.

En este sentido, Ios trabajos que ha venido realizando la IDEHN en torno a este tipo de desarrollo de aplicaciones informáticas, cobra suma importancia porque no solo genera la primer experiencia en el desarrollo de aplicaciones que utlizan información geográfica provista por geoservicios en esta región, sino que plantea el inicio de una gran gama de oportunidades de crecimiento para desarrolladores, instituciones, empresas y público en general.

Finalmente, es importante destacar que el futuro de los geoservicios estará enfocado a proporcionar información de sensores en tiempo real, a una sociedad de usuarios equipados con instrumentos de comunicación "inteligentes", que ofrecerán y demandarán información basada en su ubicación para su representación en diversos planos dimensionales. Esto pone en evidencia que aún hay mucho camino por recorrer en el campo del desarrollo de aplicaciones para dispositivos móviles, que realicen un consumo de los servicios geográficos ofrecidos por una IDE.

\section{Trabajos Futuros}

Las infraestructuras de datos espaciales, como consecuencia del cambio propiciado por las tendencias tecnológicas como son dispositivos móviles, sensores y web2.0, están convergiendo hacia sistemas móviles, en tiempo real y con participación activa de los ciudadanos. Esto vislumbra un importante desarrollo informático en torno a plataformas de manejo de información de esta índole y aplicaciones que consuman sus datos. En zonas como la Huetar Norte, temas relacionados con agricultura de precisión, en donde convergen el software y la información geográfica, plantean retos significativos en esta línea de trabajo.

Actualmente, la IDEHN trabaja en la evaluación del impacto social del geoportal, con el objetivo de identificar posibles aspectos de mejora, planteamientos metodológicos para la generación de información geográfica mediante técnicas de mapeo participativo y la incorporación del concepto de IDE semánticas sociales. También, a mediano plazo se plantean temas de Big Data, observatorios inteligentes de datos espaciales y la vinculación de la IDE con el gobierno electrónico.

Temas como éstos y otros más que se discuten, plantean retos significativos hacia futuros desarrollos tecnológicos, a partir de las soluciones existentes y también aportes significativos en temas de investigación aplicada y científica.

\section{Referencias}

[1] J. Martínez de Antoñana. Pasado, presente y futuro de las Infraestructuras de datos espaciales, 2da Edición, Bubok Publishing S.L, España, 2008. http://ww2.pcypsitna.navarra.es/Aprende/Documents/PASADOPRESENTE-Y-FUTURO-DE-LAS-INFRAESTRUCTURAS-DE-DATOS-ESPACIALES.pdf.pdf

[2] W. Kuhn, Introduction to Spatial Data Infrastructures. Institute for Geoinformatics, University of Münster, Alemania, 2005.

[3] S. Steiniger, A. Hunter, Free and Open Source GIS Software for Building a Spatial Data Infrastructure. Geospatial Free and Open Source Software in the 21st Century, 2012, pp 247-261.

[4] K. Bunzel, A. Ager, C. Schrader-Patton, Up in the air: Adventures in serving geospatial data using open source software and the cloud. Washington, D.C.: ACM, 2010.

[5] eGeoMapping, eGeoMapping. [Online] Disponible en: www.egeomapping.com/

[6] Unknown. Android Studio Overview. Recuperado el 2 de abril de 2016, de http://developer.android.com/intl/ es/tools/studio/index.html 
[7] geoserver.org. Geoserver. [Online] Disponible en: http://geoserver.org/. 2014.

[8] Leafletjs.com. Leaflet - an open-source JavaScript library for interactive maps, [Online] Disponible en: http:// leafletjs.com/. 2005.

[9] postgis.net. (2016). Spatial and geographic objects for PostgreSQL, [Online] Disponible en: http://postgis.net/

[10] postgresql.org. (2016). PostgreSQL, the world's most advanced open source data base, [Online] Disponible en: https://www. postgresql.org/

[11] OpenGIS Web Map Service (WMS) Implementation Specification 1.3.0, 06-042, 2006, [Online] Disponible en: http://www.opengeospatial.org/standards/wms.

[12] OpenGeoespatial.org (2016). OpenGIS Web Feature Service (WFS) Implementation Specification 1.1.0, 04-094, 2005, [Online] Disponible en: http://www.opengeospatial.org/standards/wfs

[13] OGC. (2016). OpenGIS Geography Markup Language (GML) Encoding Standard 3.2.1, 07-036, 2007, [Online] Disponible 